\author{
(․ Е.В. Бирюкова', А. Джаббар², С.В. Елизарова
}

'ФГБОУ ВО Московский государственный медико-стоматологический университет имени А.И. Евдокимова Минздрава

России, Москва

Eli Lilly S.A., Дубай, Объединенные Арабские Эмираты

${ }^{3} \mathrm{OОО}$ «Лилли Фарма», Москва

Достижение целей гликемического контроля посредством активной клинической тактики ведения пациентов с сахарным диабетом 2 типа (СД2) является важным условием снижения риска развития и прогрессирования связанныхс данным заболеванием осложнений. Распространенность микрососудистых и макрососудистых осложнений диабета в России имеет высокий уровень, это является следствием поздней диагностики СД, отсутствием своевременного начала терапии заболевания и не всегда тщательно подобранной сахароснижающей терапии, что подчеркивает необходимость улучшения контроля над заболеванием. Для пациентов с СД2, когда терапия пероральными сахароснижающими препаратами (ПССП) в сочетании с изменением образа жизни оказывается неэффективной для поддержания целей гликемического контроля, наиболее часто используемой опцией старта инсулинотерапии является назначение базального инсулина. Однако зачастую после начала инсулинотерапии пациенты в течение длительного периода времени остаются на терапии базальным инсулином в комбинации с ПССП, несмотря на отсутствие оптимального гликемического контроля. В данной статье мы предлагаем рассмотреть несколько вариантов стратегии интенсификации инсулинотерапии при ведении пациентов с СД2, их преимущества и недостатки. Данные подходы включают в себя добавление прандиального инсулина к терапии базальным инсулином или назначение готовых смесей (двухфазных) инсулинов. При выборе оптимальной стратегии интенсификации инсулинотерапии важно учитывать индивидуальные цели терапии, особенности образа жизни и режима питания пациентов, что позволит обеспечить успех сахароснижающей терапии.

КЛЮЧЕВЫЕ СЛОВА: сахарный диабет 2 типа; инсулин; готовые смеси инсулина; интенсификация инсулинотерапии; гликированный гемоглобин ( $\left.\mathrm{bl}_{1}\right)$; эффективность; безопасность

\title{
WHEN BASAL INSULIN IS NOT ENOUGH: SUCCESSFUL STRATEGIES FOR INSULIN INTENSIFICATION IN PATIENTS WITH TYPE 2 DIABETES MELLITUS
}

\author{
(c) Elena V. Biryukova', Abdul Jabbar², Svetlana V. Elizarova ${ }^{3}$
}

\author{
'Moscow state medical dental University named A. I. Evdokimov, Moscow, Russia \\ Eli Lilly and Company, Dubai, United Arab Emirates \\ ${ }^{3}$ Lilly Pharma Ltd, Moscow, Russia
}

Maintaining glycemic control through intensive clinical management of patients with type 2 diabetes mellitus (T2DM) is well recognized to reduce the risk of diabetes-associated complications. Patients in Russia have high rates of microvascular and macrovascular complications as a result of undiagnosed, untreated, or inadequately treated diabetes, emphasizing the need for better clinical management. The introduction of basal insulin therapy is often necessary for patients with T2DM when oral antihyperglycemic drugs and lifestyle management strategies are no longer effective inmaintaining glycemic targets. However, after initiation of insulin, patients often remain on basal insulin for long periods despite suboptimal glycemic control, and intensification of insulin therapy is frequently necessary. Here, we report on several different insulin intensification strategies available to clinicians and their patients to improve glycemic control and the advantages and disadvantages of each approach. These strategies include the use of short- and long-acting insulins administered either as bolus doses or as premixed insulins. When selecting the most appropriate intensification strategy, clinicians should consider the lifestyle and treatment goals of their patients to help ensure treatment success.

KEYWORDS: diabetes mellitus, type 2; insulin; insulin mixtures; insulin intensification; hemoglobin A; glycosylated; efficacy; safety

\section{ВВЕДЕНИЕ}

Число пациентов с сахарным диабетом (СД) 1 и 2 типа в России неуклонно растет. По данным Государственного регистра больных СД, общая численность пациентов с СД в РФ на конец 2016 г. составила 4,348 млн человек, из них около 4 млн приходится на СД 2 типа (СД2).

По оценке Международной Диабетической Федерации (IDF), в 2015 г. в России насчитывалось 11,1 млн взрослых пациентов с СД; это значит, что распространенность СД 
составляет 9,2\% среди лиц в возрасте от 20 до 79 лет [2]. По данным IDF, в России более 4,6 млн недиагностированных случаев заболевания и 186,123 случаев смерти обусловлено СД [2]. По оценкам Международной Диабетической Федерации, в России количество людей с СД может в 2-3 раза превышать официальные данные Государственного регистра больных сахарным диабетом.

В 2016 г. были опубликованы результаты национального эпидемиологического наблюдательного исследования NATION. Данные этого исследования продемонстрировали, что распространенность СД2 среди населения в возрастной категории 20-79 лет составляет 5,4\%, при этом у 2,5\% участников данного исследования СД был диагностирован ранее, а у 2,9\% респондентов - впервые. Таким образом, 54\% участников исследования имели СД2, но не знали о своем заболевании [3].

Как следствие поздней диагностики СД, отсутствия своевременного начала терапии и не всегда тщательно подобранной сахароснижающей терапии у многих пациентов развиваются опосредованные СД осложнения (диабетическая ретинопатия, почечная недостаточность, сердечно-сосудистые заболевания, синдром диабетической стопы). По данным наблюдательного исследования A1chieve, в котором были проанализированы около 67000 пациентов с СД2 в период с 2009 по 2010 гг. в 7 странах мира, в Российской Федерации были отмечены высокие показатели распространенности микрососудистых (72,4\%) и макрососудистых (89,3\%) осложнений, связанных с СД [4]. Данные национальных контрольно-эпидемиологических исследований мониторинга пациентов с СД 1 типа (СД1) и СД2 в России показали, что частота диабетической ретинопатии (38\%) и диабетической нефропатии (41\%) выше в 1,5 и 3 раза соответственно, чем официально зарегистрированные данные [5]. Согласно данным Государственного регистра СД за 2016 г., базальный инсулин является лидирующей схемой на старте инсулинотерапии, но при этом только 52,1\% пациентов с СД2 имеют уровень $\mathrm{HbA}_{1 с}$ $<7 \%$ [1]. Эти выводы свидетельствуют о необходимости повышения эффективности управления диабетом в Российской Федерации.

\section{ОБОСНОВАНИЕ ДЛЯ ПРОВЕДЕНИЯ ДАННОГО ОБЗОРА}

Тактика активного клинического ведения пациентов с СД прежде всего направлена на достижение эффективного гликемического контроля и уменьшение риска развития и прогрессирования осложнений, связанных с СД [6, 7]. Как правило, назначение инсулинотерапии рекомендуется, когда предшествующая терапия, направленная на изменение образа жизни (диета, физические нагрузки), в сочетании с приемом пероральных сахароснижающих препаратов (ПССП)/и или других инъекционных сахароснижающих препаратов неэффективна для достижения целей гликемического контроля [8]. Однако, несмотря на назначение базального инсулина, многие пациенты не достигают целевых значений гликемии и нуждаются в интенсификации инсулинотерапии, что является важнейшим условием снижения риска развития опосредованных диабетом осложнений. Целью данного обзора является предоставление данных о клинических исходах пациентов, находящихся на терапии базальным инсулином в условиях «реальной клинической практики», и стратегии интенсификации ин- сулинотерапии, в случае если терапия базальным инсулином не является эффективной.

\section{СОВРЕМЕННЫЕ РЕКОМЕНДАЦИИ ПО ИНИЦИАЦИИ ИНСУЛИНОТЕРАПИИ У ПАЦИЕНТОВ С СД2}

Вследствие прогрессирующего характера заболевания пациентам с длительным анамнезом СД2 на определенном этапе требуется и они имеют пользу от назначения инсулина [9]. Согласно рекомендациям Американской Диабетической Ассоциации (ADA) от 2017 г., назначение базального инсулина рекомендуется для пациентов, которые не достигают или не удерживают целевой уровень $\mathrm{HbA}_{1 c}$ на максимально переносимой дозе метформина \pm другие неинсулиновые сахароснижающие препараты на протяжении более 3 мес. Инсулинотерапия имеет преимущества, когда иные сахароснижающие препараты не могут быть рассмотрены в качестве комбинированной терапии при наличии выраженной гипергликемии или симптомов кетоацидоза (потеря веса, кетонурия); инсулинотерапия рекомендована также пациентам с впервые выявленным СД2, имеющим выраженные клинические симптомы заболевания и/или уровень гликированного гемоглобина $\mathrm{HbA}_{1 с} \geq 10 \%$ (86 ммоль/моль) и/или уровень глюкозы в крови $\geq 300$ мг/дл (16,7 ммоль/л) [9].

На старте инсулинотерапии цели гликемического контроля должны быть определены индивидуально для каждого пациента. При определении целей терапии необходимо учитывать несколько аспектов. Цели должны быть индивидуализированы с учетом длительности СД, возраста пациента, ожидаемой продолжительности жизни, наличия сопутствующих заболеваний, подтвержденных сердечно-сосудистых заболеваний и микрососудистых осложнений, наличия эпизодов нераспознаваемой гипогликемии и особенностей пациента. Согласно рекомендациям ADA, оптимальный уровень $\mathrm{HbA}_{1 с}$ для снижения риска развития микрососудистых осложнений составляет <7\%, однако цели терапии должны соответствовать индивидуальным потребностям каждого пациента и особенностям течения заболевания. Примечательно, что в последних рекомендациях ADA эксперты обращают внимание на роль пациента в лечении заболевания и подчеркивают, что, когда это возможно, выбор схемы терапии необходимо обсуждать с пациентом с учетом его предпочтений, потребностей и ценностей [9]. Пациентам на инсулинотерапии с нераспознаваемой гипогликемией или документально зафиксированными эпизодами выраженной гипогликемии следует установить более высокие целевые значения гликемии во избежание повтора гипогликемической реакции хотя бы на несколько недель для того, чтобы избежать повторных эпизодов гипогликемии и снизить риск развития этого осложнения в будущем [9].

\section{РОССИЙСКИЕ РЕКОМЕНДАЦИИ ПО НАЧАЛУ ИНСУЛИНОТЕРАПИИ}

Алгоритмы специализированной медицинской помощи больным СД говорят о том, что с момента установления диагноза все пациенты должны быть информированы о прогрессирующем характере течения СД2 и возможности начала лечения инсулином [10]. Инсулинотерапия показана в нескольких случаях. Это впервые выявленные 
пациенты с СД2, если уровень $\mathrm{HbA}_{1 с}$ >9\% (в этом случае инсулинотерапия может быть назначена временно); пациенты, которые не достигают индивидуальных целей терапии на оптимальных дозах ПССП/неинсулиновых препаратах или их комбинациях и в случае развития кетоацидоза. Общие рекомендации по выбору режима инсулинотерапии основываются на таких факторах, как образ жизни, особенности течения заболевания. Для пациентов, которые имеют низкую физическую активность и выражают желание делать меньше инъекций, а также не готовы к режиму многократных инъекций, есть два варианта инициации инсулинотерапии: базальный инсулин или готовые смеси инсулина. Дальнейший выбор схемы инсулинотерапии среди указанных двух режимов основывается на показателях гликемии: для пациентов с СД2, если текущий уровень $\mathrm{HbA}_{1 с}$ превышает индивидуальные целевые значения не более чем на 1-1,5\%, рекомендовано начинать терапию с базального инсулина. Если же данная разница превышает 1,5\%, то оптимальным вариантом инициации инсулинотерапии является назначение готовых смесей инсулина для коррекции гликемии натощак и после еды. Во всех остальных случаях, если у пациента с СД2 уровень $\mathrm{HbA}_{1 с}$ выше целевого более чем на 1,5\%, но он ведет активный образ жизни и мотивирован на проведение регулярного самоконтроля глюкозы крови, готов делать многократные инъекции инсулина, то в таком случае оптимальным решением будет назначение базально-болюсного режима инсулинотерапии. Цели гликемического контроля должны быть установлены индивидуально с учетом возраста пациента, отсутствия или наличия у него макрососудистых осложнений и риска развития тяжелых гипогликемических реакций [10].

\section{СТАРТ ИНСУЛИНОТЕРАПИИ У ПАЦИЕНТОВ С СД2}

Согласно Российским рекомендациям по терапии СД2, выделяют несколько стратегий старта инсулинотерапии [10]. В действительности, в реальной клинической практике назначение базального инсулина является наиболее часто используемым режимом начала инсулинотерапии, и многие пациенты могут оставаться на данном режиме на протяжении многих лет, несмотря на неудовлетворительный контроль гликемии. Ниже приводятся данные нескольких исследований, демонстрирующих результаты ведения пациентов с СД2 в ситуациях, когда пациенты длительное время оставались на стартовом режиме инсулинотерапии в условиях отсутствия достижения целей гликемического контроля.

Десятилетнее ретроспективное исследование, проводимое в Великобритании в период с 1995 по 2005 гг., в которое были включены 1513 пациентов с СД2, продемонстрировало, что большинство пациентов (73,4\%), находившихся на терапии ПССП и/или инсулине на протяжении более чем 6 мес после инициации инсулинотерапии, имели уровень $\mathrm{HbA}_{1 с} \geq 7,5 \%$ (т.е. только около 25\% пациентов достигли целей гликемического контроля). По результатам этого исследования авторы пришли к выводу, что пациенты имели низкий уровень веры в потенциальную эффективность инсулина и испытывали чувство вины за то, что вынуждены получать инсулин. Не исключено, что сами врачи могли быть причиной клинической инерции, что, в свою очередь, приводило к назначению субоптимальной терапии и отсутствию интенсификации инсулинотерапии, однако авторы говорят, что по данным этого исследования было трудно оценить истинную клиническую картину [11].

Другое ретроспективное наблюдательное исследование с участием 2417 пациентов с СД2 проводилось в США в период с 1999 по 2007 гг., в нем для наблюдения использовались данные общей базы учреждений здравоохранения. В этом исследовании пациенты наблюдались в течение 7 лет после старта инсулинотерапии. За весь этот период пациентам назначались разные препараты инсулина: в начале исследования количество пациентов на базальном инсулине составляло 68,7\% (66,8\% нейтральный протамин Хагедорна (НПХ) и 1,9\% инсулина длительного действия), в конце наблюдения - 42,5\% (36,8\% НПХ и 5,7\% инсулина длительного действия). Исследование продемонстрировало, что только от 30\% до 37\% пациентов достигли уровня $\mathrm{HbA}_{1 c}<7 \%$. Анализируя полученные результаты, авторы отметили, что наблюдательные исследования не могут выявить причину, почему так много пациентов не достигают целевых значений гликемии. Большинство пациентов боялись делать инъекции, а врачи, которые, как правило, ограничены во времени на приеме, хотели избежать тех сложностей, которые могут возникнуть при назначении больному инсулина [12].

В рандомизированном контролируемом исследовании терапии до цели (Treat-to-Target) 756 пациентов с СД2 и недостаточным контролем гликемии $\left(\mathrm{HbA}_{1 c}>7,5 \%\right)$ на терапии одним или двумя ПССП были рандомизированы в группы лечения инсулином гларгин или инсулином НПХ [13]. В данном исследовании количество пациентов, достигших целевого значения $\mathrm{HbA}_{1 c} \leq 7 \%$, было выше, чем в большинстве других исследований, но не все пациенты достигли установленных целей после 24 нед терапии; только 58,0\% в группе инсулина гларгин и 57,3\% в группе инсулина НПХ достигли целевого уровня $\mathrm{HbA}_{1 c} \leq 7 \%$. Анализируя результаты назначения базального инсулина в данном исследовании, можно выявить целый ряд моментов. Во-первых, цель по гликемии натощак была довольно низкая (100мг/дл (5,6 ммоль/л)), и, несмотря на действительно небольшое количество пациентов в группе инсулина гларгин, у кого имела место гипогликемия, баланс риск/польза мог быть барьером для успешной титрации дозы инсулина до достижения целей терапии. Во-вторых, не удалось получить ответ на некоторые важные вопросы, такие как: для каких подгрупп пациентов, получающих базальный инсулин, наиболее вероятно достижение целей терапии; становится ли пациент, перенесший гипогликемию, менее вовлеченным в титрацию дозы инсулина? Будут ли такие пациенты иметь более высокий уровень $\mathrm{HbA}_{1 c}$ ? Каким образом пациентам, не достигшим или не удерживающим целевой уровень $\mathrm{HbA}_{1 c}$ с помощью одной инъекции базального инсулина, интенсифицировать терапию с помощью добавления прандиального инсулина [13]?

В еще одном перекрестном исследовании были проанализированы 126811 пациентов с СД2 в возрасте 31-90 лет в течение 2010 г. (анализ электронной базы данных первичной медицинской помощи SIDIAPQ). Из этой когорты 9899 пациентов с СД2 в течение более 6 мес до включения в исследование получали терапию базальным инсулином. Исследование показало, что одна четверть пациентов с СД2, получающих базальный инсулин (23,5\% ( $n=2322))$, имеют трудности в достижении рекомендуемой цели $\mathrm{HbA}_{1 c^{\prime}}$ несмотря на оптимальные показатели гликемии натощак [14]. 
Большинство пациентов $(51,3 \%)$ не достигли ни одной из поставленных целей. Среди пациентов, кто не достиг рекомендуемого уровня $\mathrm{HbA}_{1 c^{\prime}}$ были те, кому удалось достичь цели по глюкозе плазмы натощак (ГПН) (<130 мг/дл). Это были пациенты более старшей возрастной категории, имевшие более длительный анамнез СД, изначально более низкие значения $\mathrm{HbA}_{1 c^{\prime}}$ индекса массы тела (ИМТ), диастолического артериального давления (ДАД), общего холестерина и липопротеидов низкой плотности (ЛПНП), чем те пациенты, кто не достиг целей по ГПН. У пациентов, у которых НbА чала терапии базальным инсулином, вклад колебаний постпрандиальной гликемии (ППГ) в общую гипергликемию составлял более 60\%. Авторы констатируют, что у пациентов, которые добросовестно титруют базальный инсулин, именно ППГ определяет постоянно повышенный уровнь $\mathrm{HbA}_{1 c^{\prime}}$ независимо от уровня ГПН. Данный факт говорит о том, что пациенты, получающие базальный инсулин, могут быть некомпенсированы и что необходимые изменения тактики инсулинотерапии, такие как интенсификация, иногда происходят после нескольких лет неадекватного гликемического контроля. Руководства по терапии СД рекомендуют раннее назначение сахароснижающей терапии и своевременное назначение комбинации препаратов для улучшения контроля гликемии, но врачи обычно предпочитают поэтапный подход к лечению больных, вследствие которого пациент зачастую проводит более 10 лет с уровнем $\mathrm{HbA}_{1 c}>7 \%$ (53 ммоль/моль) и 5 лет с уровнем $\mathrm{HbA}_{1 c}>8 \%$ (64 ммоль/моль), прежде чем доктор назначит инсулин. В этом исследовании восприятие пациентами уровня вовлеченности и внимательности врача, качество объяснения данных лабораторного исследования и интерпретация их результатов, а также обсуждение необходимого режима терапии были непосредственно связаны с поведением пациентов в отношении соблюдения ими рекомендованного режима инсулинотерапии [14].

Ниже приведены данные исследований, в которых оценивались различные подходы для инициации инсулинотерапии у пациентов с СД2, не достигающих целевых показателей гликемического контроля на терапии ПССП.

В исследовании INITIATE 233 пациента с СД2 и недостаточным контролем гликемии на монотерапии метформином или в комбинации с другими ПССП были рандомизированы в группы терапии инсулином гларгин или двухфазным инсулином аспарт 30/70 (30\% инсулина аспарт и 70\% протаминированной суспензии инсулина аспарт) [15]. После 28 нед терапии 66\% пациентов в группе терапии двухфазным инсулином аспарт 30/70 и 40\% пациентов в группе терапии инсулином гларгин достигли целей терапии $\mathrm{HbA}_{1 c}<7$. Несмотря на то что в группе пациентов, получающих инсулин аспарт 30/70, отмечалось большее число эпизодов гипогликемии легкой степени (эпизодов/год) в сравнении с группой инсулина гларгин (3,4 46,6 и 0,7 $\pm 2,0$ соответственно; $p<0,05)$, а также увеличение массы тела и суточной дозы инсулина в конце наблюдения было больше у пациентов в группе аспарт 30/70 в сравнении с группой инсулина гларгин (увеличение

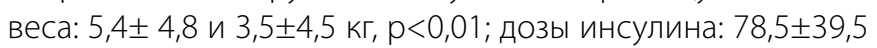
и 51,3 26,7 Ед/сут соответственно), двухфазный инсулин аспарт 70/30 продемонстрировал бо́льшую эффективность в сравнении с инсулином гларгин по снижению уровня $\mathrm{HbA}_{1 с}$ у пациентов, которые были включены в исследова- ние $\mathrm{c} \mathrm{HbA}_{1 с} \geq 8,5 \%$. Это подтверждает факт того, что по мере снижения функции бета-клеток и с увеличением уровня $\mathrm{HbA}_{1 c}$ назначения только базального инсулина недостаточно, поскольку необходим контроль постпрандиальной гликемии [15].

В ретроспективном наблюдательном исследовании, проведенном в Великобритании в течение 36 мес, были проанализировали данные базы The Health Improvement Network (THIN), собранные с 2002 по 2006 гг. [16]. В исследование были включены 8009 пациентов с СД2 с неудовлетворительным контролем гликемии (исходный (среднее-

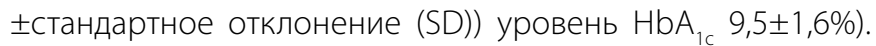
Пациенты получали терапию ПССП и/или рекомендации по изменению образа жизни и им был назначен базальный инсулин (НПХ, детемир или гларгин) или готовые смеси инсулина, которые они получали в течение всего периода наблюдения. Хотя все режимы инсулинотерапии привели к значимому снижению $(\mathrm{p}<0,001)$ уровня $\mathrm{HbA}_{1 с}$ через 12 нед терапии, среднее значение $( \pm \mathrm{SD}) \mathrm{HbA}_{1 с}(8,4 \pm 1,5 \%)$ не соответствовало рекомендуемым показателям. Несмотря на то что целевые значения гликемии достигнуты не были, 75\% пациентов продолжали терапию инсулинами НПХ, 78\% - инсулином детемир, 83\% - инсулином гларгин и 92\% - готовой смесью инсулина в течение первых 12 мес исследования. Через 36 мес пациенты, которые получали готовую смесь инсулина, смогли дольше всего оставаться на данной терапии (83\%), далее следуют инсулин гларгин (67\%) и НПХ (57\%). (Примечание: данные после 36 мес наблюдения были некорректны для инсулина детемир, так как он был зарегистрирован в Великобритании позже, чем другие инсулины.) [16].

В другом ретроспективном наблюдательном исследовании, проведенном в Великобритании с помощью базы THIN, были собраны данные за период с 2004 по 2006 гг. с целью проанализировать подходы к инициации инсулинотерапии у пациентов с СД2 и неудовлетворительным гликемическим контролем (средний уровень $\mathrm{HbA}_{1 с}( \pm \mathrm{SD})$ 9,6士2,0\%)) [17]. Из 4045 пациентов, включенных в исследование, 52,4\% был назначен базальный инсулин, 41,6\% готовая смесь инсулина, 4,0\% - базально-болюсный режим и 2,1\% - только прандиальный инсулин. После 6 мес терапии у пациентов, чей средний ( $\pm \mathrm{SD}$ ) уровень $\mathrm{HbA}_{1 с}$

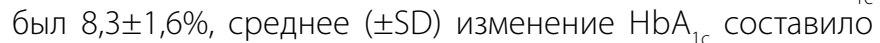

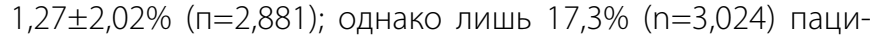
ентов достигли уровня $\mathrm{HbA}_{1 c}<7 \%$. В целом по истечении 6 мес наблюдения из всех включенных в исследование пациентов 75,1\% остались на прежней схеме инсулинотерапии, 13,7\% пациентов отменили инсулин, в 7,0\% случаев был назначен другой режим терапии и у 4,7\% была интенсифицирована инсулинотерапия. Авторы данного исследования указали на две проблемы, связанные с назначением инсулина, - увеличение массы тела и риск развития гипогликемии и подчеркнули, что, по данным клинических исследований, среднее увеличение массы тела у пациентов с СД2 составило до 3 кг за 6 мес после инициации инсулинотерапии в комбинации с ПССП, хотя увеличение веса могло отличаться в зависимости от вида инсулина [17].

В 2015 г. были опубликованы данные многоцентрового наблюдательного исследования MOSA1c с участием 4341 пациента из 18 стран мира. В этом исследовании проводился анализ применения инсулина в терапии пациентов с СД2 в разных странах и изучались основные барьеры, 
связанные с интенсификацией инсулинотерапии, возникающие как со стороны врачей, так и пациентов и системы здравоохранения. Данное исследование показало, что подходы к инициации инсулинотерапии различаются в разных странах: в Германии на старте инсулинотерапии с одинаковой частотой назначается как базальный инсулин, так и готовые смеси инсулинов (44\% и 44\%); в Китае 16\% пациентов получают в качестве стартового режима инсулинотерапии базальный инсулин и 66\% - готовые смеси инсулина; в США ситуация прямо противоположная, в 66\% случаев назначается базальный инсулин и в 17\% - готовые смеси инсулина. Россия является лидером по стратегии инициации терапии с помощью базального инсулина, 82\% пациентов начинают инсулинотерапию с базального инсулина и только 8\% пациентам на старте назначаются готовые смеси инсулина [18]. В данное исследование включали пациентов, которые получали инсулинотерапию на протяжении более 3 мес за исключением пациентов на базис-болюсной схеме инсулинотерапии. Исследователи обратили внимание на разницу между уровнем $\mathrm{HbA}_{1 с}$ на момент включения пациентов в исследование и целью терапии, установленной врачом; подавляющее большинство пациентов, несмотря на проводившуюся им инсулинотерапию, не достигли установленного целевого значения гликемии. Исследование MOSA1с также продемонстрировало, что существует взаимосвязь между тем, как пациенты воспринимают взаимодействие с врачом, и беспокойством, которое они испытывают по поводу своего заболевания. Оба эти фактора: и взаимоотношение с врачом, и эмоциональное состояние пациента с СД напрямую влияют на приверженность к назначенной схеме инсулинотерапии и достижение целей гликемического контроля [18].

Стратегии терапии и тенденции в инициации и интенсификации инсулинотерапии среди пациентов с СД2 в США были оценены в ретроспективном наблюдательном исследовании с использованием базы данных частной страховой медицины [19]. В анализ были включены в общей сложности 7932 пациента, которым была инициирована терапия инсулином в период между 2003 и 2008 гг. [19]. Исходя из назначенного стартового режима инсулинотерапии группы распределились следующим образом: 61\% пациентов получали терапию базальным инсулином, 14\% готовыми смесями инсулинов, 13\% - режим базис-болюсной терапии, 11\% - только прандиальный инсулин и 1\% - комбинацию прандиального инсулина и готовой смеси инсулинов, базального инсулина и готовой смеси инсулинов или все вида 3 инсулина. Из 5570 пациентов через 6 мес после начала инсулинотерапии только в 38,1\% случаев проводилась интенсификация терапии. У 22,9\% пациентов терапия была оптимизирована путем увеличения доз или кратности приема инсулина, у 5,7\% был добавлен прандиальный инсулин и 1,2\% - добавлена готовая смесь инсулина. В ходе исследования 11,8\% пациентов прекратили прием всех сахароснижающих препаратов и 10,0\% пациентов остались на терапии неинсулиновыми препаратами. Частота случаев проведения интенсификации инсулинотерапии и прекращения терапии инсулином были сопоставимы у пациентов с разными стартовыми режимами (базальный инсулин, готовые смеси инсулинов или базис-болюсный режим инсулинотерапии). Низкие темпы интенсификации терапии и высокая частота прекращения терапии инсулином, наблюдаемая в данном исследовании, свидетельствуют, что пациенты остаются в группе риска неадекватного гликемического контроля после старта инсулинотерапии [19].

Результаты данных наблюдательных исследований отражают реальную клиническую практику инсулинотерапии и показывают, что примерно от 5\% до 40\% пациентов с недостаточным гликемическим контролем нуждаются в проведении интенсификации инсулинотерапии. Эти данные соотносятся с выводами, представленными ранее, что примерно от 30\% до 70\% пациентов, кому была инициирована терапия базальным инсулином, не достигают целей гликемического контроля и при этом остаются на данной терапии на протяжении от 6 до 12 мес, несмотря на неудовлетворительный контроль гликемии. В то же время, есть и другая схема инициации инсулинотерапии - готовые смеси инсулина, которые имеют ряд преимуществ в сравнении с базальным инсулином. В России эта стратегия используется редко, несмотря на рекомендации российских Алгоритмов специализированной медицинской помощи больным сахарным диабетом. Вместе с тем приведенные выше данные свидетельствуют о том, что большинство пациентов с СД2 для достижения целей гликемического контроля нуждаются в интенсификации инсулинотерапии.

Почему же пациенты с СД2 остаются на первоначальной схеме инсулинотерапии, несмотря на то, что они не достигают индивидуальных целей лечения? Этот вопрос обсуждается в течение многих лет. Одной из основных причин недостижения целей терапии является «клиническая инерция». Это термин, определяемый как «отказ медицинских работников инициировать или интенсифицировать терапию, когда это показано» [20]. Понятие клинической инерции комплексное, причинами могут быть барьеры на уровне врача, пациента и системы здравоохранения. Барьеры со стороны врачей связаны с ограничением времени, трудностью выбора необходимой терапии в условиях существования различных клинических рекомендаций, страха потенциального риска развития гипогликемии. На уровне пациента наиболее частыми барьерами являются низкая приверженность к проводимой терапии, страх гипогликемии и увеличения массы тела. Со стороны системы здравоохранения барьерами может быть высокая стоимость терапии новыми лекарственными препаратами и в целом высокие затраты на лекарственное обеспечение. Исследования подчеркивают, что феномен клинической инерции - это постоянная и реально существующая проблема, несмотря на наличие ясных клинических рекомендаций с четко прописанными целями терапии. Внедрение в практику этих рекомендаций могло бы быть ценным как начальная ступень в принятии клинического решения, однако практика показывает, что клиническая инерция существенно не меняется с годами, несмотря на убедительные доказательства преимуществ жесткого гликемического контроля [20].

\section{СТРАТЕГИИ ИНТЕНСИФИКАЦИИ ИНСУЛИНОТЕРАПИИ}

Согласно клиническим рекомендациям по терапии СД2, проведение интенсификации инсулинотерапии показано, если на фоне терапии базальным инсулином значения $\mathrm{HbA}_{1 c}$ остаются выше целевого уровня на протяжении от 3 до 6 мес и более или при наличии значительных колебаний ППГ (>10 ммоль/л) [8, 9, 10]. Одним из вариантов 
интенсификации сахароснижающей терапии, согласно современным клиническим рекомендациям по ведению пациентов с СД2, является добавление к базальному инсулину препаратов класса агонистов глюкагоноподобного пептида (аГПП-1), но эта стратегия не будет рассматриваться в данном обзоре, т. к. заслуживает отдельного обсуждения. К сожалению, в настоящее время использование этого класса сахароснижающих препаратов в России невелико и составляет не более 1\% от всех сахароснижающих препаратов, что может быть связано с рядом ограничений, таких как их стоимость и отсутствие широкой возможности выписки по льготному обеспечению.

Существуют различные подходы к проведению интенсификации инсулинотерапии у пациентов с СД2: (1) добавление к терапии базальным инсулином одной инъекции аналога инсулина ультракороткого действия (инсулина лизпро, аспарт или глулизин) перед максимальным приемом пищи или перед завтраком (схема базал плюс) [21]; (2) последовательное добавление к терапии базальным инсулином 2 и более инъекций прандиального инсулина перед основными приемами пищи (базис-болюсный режим терапии) [22] или (3) назначение готовых смесей инсулина с разным соотношением базального и прандиального компонентов (готовая смесь инсулина лизпро 75/25 (Хумалог Микс 25), готовая смесь инсулина лизпро 50/50 (Хумалог Микс 50), готовая смесь инсулина аспарт 70/30) обычно в режиме двукратного введения перед завтраком и ужином или 3 раза в день перед завтраком, обедом и ужином [23].

При выборе стратегии интенсификации инсулинотерапии как врачами, так и пациентами должны учитываться несколько факторов, а именно, цели терапии, включая $\mathrm{HbA}_{1 с}$ и ППГ, возраст пациента, длительность диабета, наличие или отсутствие эпизодов гипогликемии, другие сопутствующие заболевания. Предпочтения пациентов, степень сложности режима инсулинотерапии, образ жизни, уровень образования и мотивации пациента к лечению, а также психосоциальные факторы являются важными аспектами, определяющими выбор соответствующей стратегии интенсификации инсулинотерапии $[9,10]$. Эффективность проведения интенсификации инсулинотерапии определяется не только выбором стратегии терапии, но и адекватностью подобранных доз инсулина, при этом проводится титрация как базального, так и прандиального компонентов, основываясь на значениях гликемии по данным дневника самоконтроля. В большинстве случаев терапия ПССП может быть продолжена, однако при добавлении прандиального инсулина прием производных сульфонилмочевины и меглитинидов (т.е. секретагогов) обычно следует прекратить, кроме того, необходимо рассмотреть целесообразность продолжения приема препаратов класса аГПП-1 при переводе пациента на базис-болюсный режим [10].

\section{ПРЕИМУЩЕСТВА И НЕДОСТАТКИ СТРАТЕГИЙ ИНТЕНСИФИКАЦИИ ИНСУЛИНОТЕРАПИИ}

\section{Режим базал плюс и базис-болюсная терапия}

Режим «базал плюс» (базальный инсулин плюс одна инъекция прандиального инсулина) применяется для последовательной интенсификации инсулинотерапии у пациентов с СД2, которые не достигают оптимального контроля гликемии на терапии базальным инсулином, что позволяет улучшить гликемический контроль без назначения полно- го (интенсивного) режима базис-болюсной терапии [21]. Для тех пациентов, которым не удается достичь целей гликемического контроля на режиме базал плюс, может потребоваться назначение дополнительных инъекций прандиального инсулина (базис-болюсный режим терапии). Стратегия базис-болюсной инсулинотерапии позволяет наиболее точно имитировать физиологические колебания гликемии, при этом титрация базального и прандиального компонентов данного режима терапии может проводиться независимо друг от друга, обеспечивая оптимальный гликемический контроль как гликемии натощак, так и после приема пищи.

Базис-болюсный режим инсулинотерапии обеспечивает большую гибкость терапии относительно времени введения прандиального инсулина и, следовательно, больший потенциал для контроля ППГ по сравнению с терапией готовыми смесями инсулина [7]. Однако в настоящее время не существует международных рекомендаций, которые бы утверждали преимущества использования в клинической практике базис-болюсного режима терапии перед другими схемами интенсификации инсулинотерапии [24]. Базис-болюсная терапия - более сложный режим, чем терапия готовыми смесями инсулина или терапия с помощью базального инсулина, и требует дополнительного обучения пациентов проведению самоконтроля гликемии, методике коррекции доз прандиального инсулина и количественному определению углеводов в пище. Ниже представлены результаты трех рандомизированных клинических исследований, в которых изучались стратегии добавления прандиального инсулина в виде схемы базал плюс или базис-болюсной терапии пациентам, ранее получавшим терапию базальным инсулином.

\section{Исследование FullSTEP}

Исследование FullSTEP является одним из исследований, в котором на примере применения ультракороткого инсулина аспарт было продемонстрировано, что интенсификация инсулинотерапии путем ступенчатого добавления прандиального инсулина к терапии базальным инсулином (детемир) обеспечивает сопоставимый гликемический контроль в сравнении с назначением полного режима базис-болюсной терапии инсулинами аспарт и детемир [22]. Обе стратегии продемонстрировали свою эффективность в улучшении гликемического контроля; однако терапия в режиме базал плюс сопровождалась достоверно меньшей частотой развития гипогликемических реакций в сравнении с полным базально-болюсным режимом. Низкая частота гипогликемий объясняется как результат последовательного добавления инъекций прандиального инсулина, что позволяет пациентам адаптироваться к режиму проведения самоконтроля гликемии и титрации доз прандиального инсулина аспарт.

В исследовании FullSTEP также была отмечена большая удовлетворенность пациентов лечением в группе терапии пошагового добавления прандиального инсулина; возможно, это было связано с меньшим количеством инъекций (2 инъекции в сравнении с 4 инъекциями) и меньшим количеством точек самоконтроля у пациентов, которым потребовалось введение 1 или 2 инъекций прандиального инсулина, в сравнении с полным базис-болюсным режимом терапии. Всем пациентам в исследовании FullSTEP перед добавлением инъекций прандиального инсулина 
были подобраны оптимальные дозы базального инсулина детемир; кроме того, в исследовании использовался упрощенный алгоритм титрации прандиального инсулина «1-0-1», чтобы повысить приверженность пациентов к проведению самостоятельной титрации доз прандиального инсулина $[22,25]$. Результаты данного исследования показали, что использование в клинической практике стратегии последовательного добавления прандиального инсулина позволяет уменьшить риск развития гипогликемических реакций, увеличения веса, а также упростить терапию прандиальным инсулином, улучшив соблюдение терапевтического режима пациентами в сравнении с назначением базис-болюсной терапии в полном режиме [22].

\section{Исследование AUTONOMY}

Исследование AUTONOMY на примере использования инсулина лизпро продемонстрировало, что инсулин ультракороткого действия может эффективно и безопасно добавляться к терапии базальным инсулином гларгин с использованием двух разных алгоритмов титрации, проводимых пациентами самостоятельно, ежедневно [Q1D] или раз в три дня [Q3D]. В данном исследовании первая инъекция прандиального инсулина вводилась перед завтраком и в дальнейшем, при необходимости, пациентам добавлялась 2-я или 3-я инъекция перед обедом и ужином, титрация осуществлялась по уровню гликемии перед следующим приемом пищи. Результаты этого исследования согласуются с ранее представленными данными [26] о том, что стратегия постепенного добавления прандиального инсулина к базальному может безопасно использоваться у категории пожилых пациентов с СД2, поскольку в исследовании AUTONOMY в подгруппе пациентов $\geq 65$ лет инициация терапии прандиальным инсулином не продемонстрировала повышения риска развития гипогликемических реакций [24]. В данном исследовании у пациентов имело место увеличение массы тела независимо от того, какой алгоритм титрации использовался; однако в группе пациентов, проводивших титрацию инсулина раз в три дня (Q3D), была отмечена несколько большая прибавка массы тела. Важным аспектом исследования AUTONOMY было наличие периода оптимизации доз инсулина гларгин перед добавлением прандиального инсулина лизпро. Для любой стратегии интенсификации инсулинотерапии с использованием базис-болюсного подхода перед добавлением инъекций прандиального инсулина необходимо убедиться в эффективности дозы базального инсулина и наличии оптимального контроля уровня гликемии натощак [24].

\section{Исследование START}

Исследование START, в котором в качестве прандиального инсулина использовался аналог инсулина ультракороткого действия глулизин, показало, что проведение титрации прандиального инсулина пациентами самостоятельно с использованием простого алгоритма было так же эффективно, как проведение титрации под руководством врача [27]. Самостоятельная титрация инсулина пациентами была сопоставима с проведением титрации под руководством врача по уровню снижения $\mathrm{HbA}_{1 с}$ в конце исследования [27]. Было отмечено статистически значимое увеличение массы тела в обеих группах терапии по сравнению с исходными показателями, при достоверно большей динамике веса в группе титрации прандиального инсулина пациентами самостоятельно в сравнении с группой титрации под врачебным руководством. Не было отмечено разницы в частоте гипогликемических реакций между группами терапии [27].

В исследовании START продемонстрирован простой и удобный подход добавления прандиального инсулина перед завтраком, что помогает повысить приверженность пациентов терапии. Кроме того, такая схема безопасна для пациента за счет снижения риска ночной гипогликемии. В этой стратегии также использованы общие принципы самостоятельного измерения глюкозы крови натощак перед завтраком, минимизируя таким образом потребность в дополнительных точках самоконтроля в течение дня. Еще одним преимуществом данного подхода является удобство выполнения инъекции в домашних условиях, что позволяет пациентам контролировать гликемию натощак утром с целью улучшения гликемического контроля в течение дня [26]. Как и в исследованиях AUTONOMY и FUIISTEP, в исследовании START пациентам перед добавлением инъекций прандиального инсулина были оптимизированы дозы базального инсулина [27].

\section{Готовые смеси инсулина}

Применение готовых смесей инсулинов является альтернативным вариантом интенсификации инсулинотерапии в ситуациях, когда другие режимы терапии (базальный инсулин, режим базал плюс или базис-болюсный режим) не обеспечивают эффективного контроля гликемии или являются неприемлемыми для пациентов. Каждая доза готовой смеси инсулина содержит в себе как базальный, так и прандиальный компонент; обычно двухфазные инсулины назначают дважды в день - одну инъекцию перед завтраком и одну перед ужином, либо трижды в день - перед завтраком, обедом и ужином [10]. Учитывая фиксированное соотношение базального и прандиального компонентов двухфазных инсулинов, данная стратегия интенсификации может быть менее гибкой, однако терапия готовыми смесями инсулина может быть оптимальным вариантом интенсификации для тех пациентов, для которых использование более сложных стратегий терапии (например, базис-болюсный режим) не приемлемо из-за необходимости выполнения многократных инъекций и проведения частого самоконтроля гликемии [10].

В настоящее время в международных руководствах по терапии Сд2 нет четких рекомендаций, для каких пациентов назначение готовых смесей инсулина будет наиболее подходящим вариантом интенсификации терапии. Одним из преимуществ терапии готовыми смесями инсулина является простота их использования - меньшее количество инъекций и требуемая частота проведения самоконтроля гликемии по сравнению с базис-болюсной стратегией терапии [8, 9, 10]. В систематическом обзоре сравнения готовых смесей аналогов инсулина и базальных аналогов инсулина терапия готовыми смесями инсулинов в режиме 2- или 3-кратного введения демонстрирует лучший гликемический контроль в сравнении с терапией базальными аналогами, вводимыми 1 раз в день [28]. В данном обзоре было показано, что при применении готовых смесей инсулина в режиме 3 инъекций в день имеет место большая общая частота гипогликемических реакций и большее увеличение массы тела в сравнении с терапией 
Старт: 10 ЕД/Кг/день

Титрация: 10-15\% или 2-4 ЕД 1-2 раза в неделю до достижения целевого уровня ГПН

В случае гипогликемии: выяснить причину и принять меры по ее устранению,

если причина не ясна, снизить дозу на 4 ЕД или 10-20\%

Если целевой уровень $\mathrm{HbA}_{1 c}$ не достигнут на стартовом режиме ИТ, рассмотреть назначение комбинации инъекционных препаратов

Добавление 1 инъекции инсулина

короткого действия перед самым

большим приемом пищи

Старт: 4 ЕД/кг или 10\% суточной дозы базального инсулина. Если $\mathrm{HbA}_{1 c}<8 \%$ суточная доза базального инсулина должна быть снижена на 10\%

Титрация: увеличение дозы на 1-2 ЕД или 10-15\% один или два раза в неделю до достижения целевых значений гликемии по данным самостоятельного мониторинга уровня глюкозы крови

Гипогликемия: определение причины; если причина не установлена, необходимо снизить соответствующую дозу на 2-4 ЕД или 10-20\%

Если целевой уровень $\mathrm{HbA}_{1 \mathrm{c}}$ не достигнут, перевод на базальноболюсный режим

\section{Добавление $\geq 2$ инъекций инсулина} короткого действия перед приемом пищи (базал-болюс)

Старт: 4 ЕД/кг или 10\% суточной дозы базального инсулина перед каждым приемом пищи. Если $\mathrm{HbA}_{1 c}<8 \%-$ суточная доза базального инсулина должна быть снижена на 10\%

Титрация: увеличение дозы на 1-2 ЕД или 10-15\% один или два раза в неделю до достижения целевых значений гликемии по данным самостоятельного мониторинга уровня глюкозы крови

Гипогликемия: определение причины; если причина не установлена, необходимо снизить соответствующую дозу на 2-4 ЕД или 10-20\%

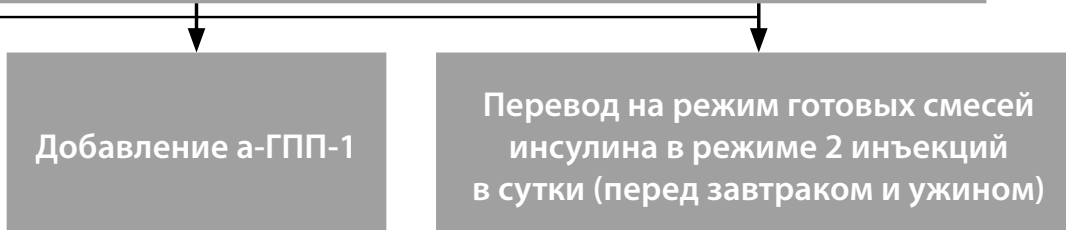

Если целевой уровень $\mathrm{HbA}_{1 \mathrm{c}}$ не достигнут или есть непереносимость $\phi$-ГПП-1, переход на ИТ в режиме

2 инъекций

Если цели терапии не достигнуты, рассмотреть перевод на альтернативные режимы инсулинотерапии

\section{Если цели терапии не достигнуты, рассмотреть перевод на альтернативные режимы инсулинотерапии}

Старт: суточная доза базального инсулина делится на: 2/3 перед завтраком и 1/3 перед ужином или $1 / 2$ перед завтраком и 1/2 перед ужином Титрация: увеличение дозы на 1-2 ЕД или 10-15\% один или два раза в неделю до достижения целевых значений гликемии по данным самостоятельного мониторинга уровня глюкозы крови

Гипогликемия: определение причины; если причина не установлена,

необходимо снизить соответствующую дозу на 2-4 ЕД или 10-20\%

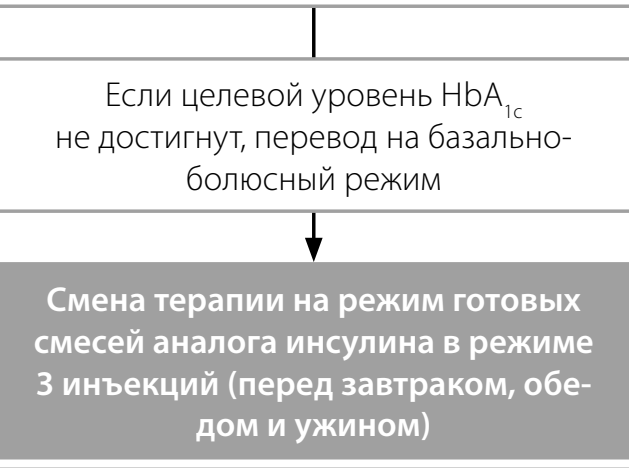

Старт: добавьте инъекцию перед обедом

Титрация: увеличение дозы на 1-2 ЕД или 10-15\% один или два раза в неделю до достижения целевых значений гликемии по данным самостоятельного мониторинга уровня глюкозы крови

Гипогликемия: определение причины; если причина не установлена, необходимо снизить соответствующую дозу на 2-4 ЕД или 10-20\%

Рис. 1. Рекомендации Американской Диабетической Ассоциации по началу и интенсификации инсулинотерапии при СД2, 2017 г. 


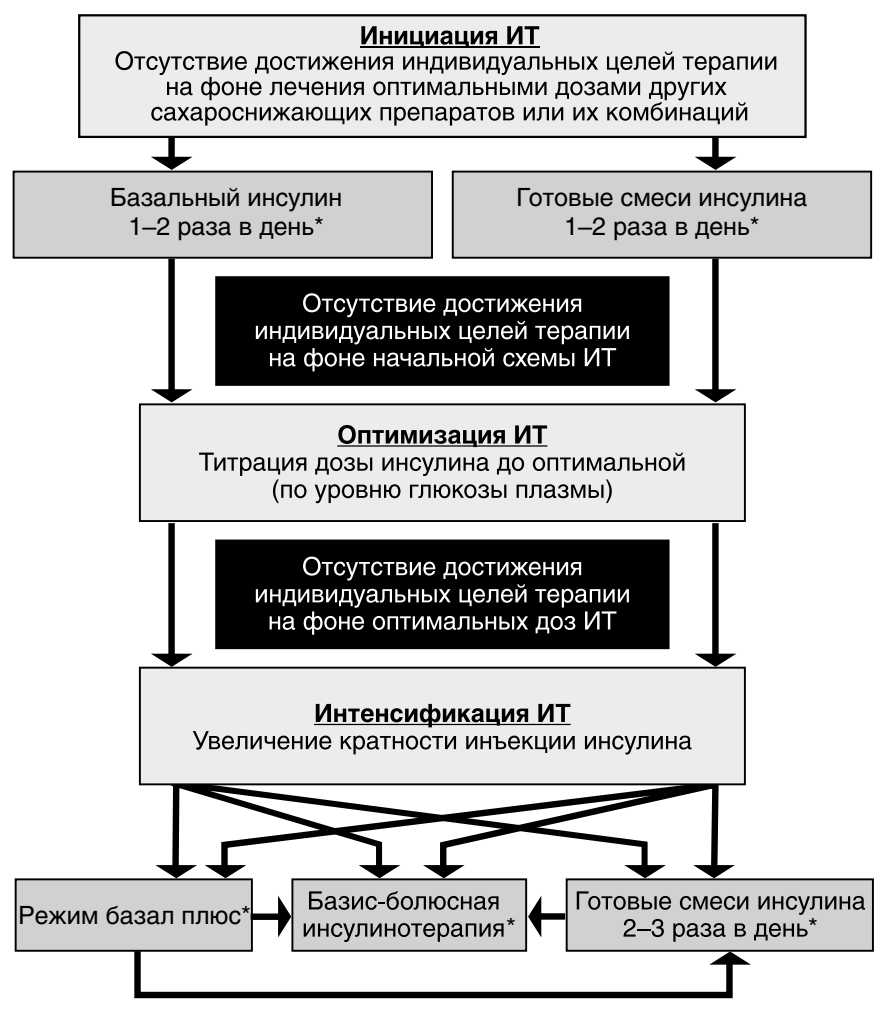

Рис. 2. Российские рекомендации по началу, оптимизации и интенсификации инсулинотерапии при СД2, 2017 год.

базальным инсулином [28]. После этого систематического обзора были проведены два других рандомизированных клинических исследования (РКИ), в которых сравнивались эффективность и безопасность терапии готовыми смесями инсулинов с базис-болюсным режимом терапии; эти исследования описаны ниже.

В исследовании Rosenstock et al. [23] проводилось сравнение терапии готовой смеси инсулина Хумалог Микс 50 с базис-болюсным режимом терапии инсулином гларгин и инсулином лизпро у пациентов с СД2 и недостаточным контролем гликемии на предшествующей терапии базальным инсулином. Инсулин Хумалог Микс 50 назначался пациентам 3 раза в сутки перед основными приемами пищи; базальный инсулин гларгин вводился перед сном, инсулин лизпро 3 раза в сутки перед едой (пациенты в группе терапии Хумалог Микс 50 получали 3 инъекции в день против 4 инъекций в день в группе базис-болюсной терапии). После 24 нед терапии обе схемы инсулинотерапии продемонстрировали сравнимую эффективность, около 80\% пациентов в обеих группах терапии достигли уровня $\mathrm{HbA}_{1 c} \leq 7,5 \%$, число пациентов, имевших $\mathrm{HbA}_{1 с}<7 \%$, было больше в группе базис-болюсной терапии. Оба режима продемонстрировали сопоставимое умеренное увеличение массы тела; у большинства пациентов в обеих группах терапии наблюдался хотя бы 1 эпизод самостоятельно зарегистрированной гипогликемии, однако частота тяжелой гипогликемии была очень низкой и не различалась между группами терапии [23].

В исследовании Tinahones et al. [29] проводилось сравнение терапии готовой смесью Хумалог Микс 25 и базальным инсулином гларгин плюс одна инъекция прандиального инсулина лизпро у пациентов с СД2 и недостаточным гликемическим контролем на предыдущей терапии инсулином гларгин в комбинации с метформином и/или пиоглитазоном. Пациенты в группе терапии инсулином Хумалог Микс 25 вводили инсулин дважды в сутки ежедневно перед завтраком и ужином; в группе терапии базал плюс базальный инсулин гларгин вводился перед сном, а инъекция инсулина лизпро - перед максимальным приемом пищи. Таким образом, общее количество ежедневных инъекций инсулина было одинаковым и составляло 2 для каждой группы терапии. После 24 нед терапии продемонстрирована не меньшая эффективность режима терапии инсулином Хумалог Микс 25 в сравнении с терапией гларгин плюс одна инъекция инсулина лизпро относительно динамики уровня НbА мо большей динамике $\mathrm{HbA}_{1 c^{\prime}}$ отмеченной в группе терапии инсулином Хумалог Микс 25; число пациентов, достигших целевого значения $\mathrm{HbA}_{1 c}<7 \%$, было сравнимо между двумя группами терапии. Как и в сравнительном исследовании инсулина Хумалог Микс 50 и терапии гларгин плюс лизпро, в данном исследовании не было отмечено значимых различий в частоте гипогликемических реакций между терапией инсулином Хумалог Микс 25 и гларгин плюс одна инъекция лизпро, однако в этом исследовании на терапии инсулином Хумалог Микс 25 была отмечена немного большая прибавка массы тела [29].

В недавнем обзоре, включавшем 15 исследований сравнения базально-болюсного режима (количество инъекций прандиального инсулина $\leq 3$ инъекции в сутки) и режима терапии готовой смесью инсулинов ( $\leq 3$ инъекции в сутки), клинически значимое преимущество режима базал-болюсной терапии по контролю гликемии было продемонстрировано только в 4 исследованиях. Только в 3 исследованиях из 15 была продемонстрирована разница в частоте развития гипогликемических реакций; в одном из которых более безопасной показала себя терапия готовой смесью инсулина, и в двух - базально-болюсная терапия. Таким образом, при выборе тактики терапии необходимо применять индивидуальный подход к интенсификации инсулинотерапии, учитывая преимущества и недостатки каждой стратегии, а также отношение и предпочтения каждого пациента - только в этом случае и режим базально-болюсной терапии, и назначение готовых смесей инсулина будет эффективным [30].

Результаты исследований по выбору режима базал плюс, базально-болюсной терапии или готовых смесей инсулина для интенсификации терапии продемонстрировали, что эти схемы являются эффективными в снижении $\mathrm{HbA}_{1 с}$ и ГПН. Кроме того, все эти режимы сопоставимы между собой по профилю безопасности, однако если увеличение массы тела может быть больше у пациентов, получающих готовые смеси инсулинов в сравнении с базальным инсулином, то при сравнении с базально-болюсным режимом разницы в динамике массы тела не наблюдалось.

На основании большой доказательной базы использования готовых смесей инсулина в терапии пациентов с СД2 в рекомендации ADA от 2017 г. были внесены следующие изменения.

Если пациент на терапии с помощью 2 инъекций готовой смеси инсулина имеет уровень $\mathrm{HbA}_{1 с}$ выше целевого значения, необходимо рассмотреть вопрос о переходе на режим готовой смеси инсулина три инъекции в день (аспарт микс 70/30, инсулин лизпро микс 75/25 или 50/50). В целом готовые смеси инсулина в режиме 3 инъекций сравнимы по эффективности с режимом базально-болюсной терапии и имеют равную частоту развития гипогликемических реакций [30]. 
Если пациент не достигает целевого уровня $\mathrm{HbA}_{1 с}$ на терапии базальный инсулин + 1 инъекция прандиального инсулина перед самым большим приемом пищи, необходимо рассмотреть возможность назначения базально-болюсной терапии с $\geq 2$ инъекциями прандиального инсулина перед приемами пищи.

Также рекомендации АДА в их последней редакции рекомендуют рассматривать вопрос о переводе пациента с одного режима терапии на другой (например, с готовой смеси инсулина три раза в день на базально-болюсный режим или наоборот), если не удается достичь целевых показателей $\mathrm{HbA}_{1 с}$ и/или в зависимости от других особенностей пациента (рис. 1 [30, 31, 32]).

В 2017 г. также были внесены обновления в российские Алгоритмы специализированной медицинской помощи больным сахарным диабетом, добавлена новая опция для интенсификации инсулинотерапии. Если пациенты не достигают индивидуальных целей на терапии в режиме базал плюс, возможен перевод их на терапию готовыми смесями инсулинов (рис. 2 [10]).

\section{ЗАКЛЮЧЕНИЕ}

По данным наблюдательных клинических исследований, отражающих реальную клиническую практику терапии пациентов с СД2, установлено, что от 1/3 до 2/3 пациентов, находящихся на терапии базальным инсулином, не достигают целей гликемического контроля, рекомендованных в международных руководствах по терапии пациентов с СД2. Пациенты на протяжении многих лет могут оставаться на данной терапии и, следовательно, иметь риск развития и прогрессирования микро- и макрососудистых осложнений. Последние эпидемиологические данные в России подчеркивают необходимость внедрения в клиническую практику эффективных стратегий управления СД с целью уменьшения риска развития сосудистых осложнений, связанных с недостаточным контролем гликемии.

На начальном этапе инсулинотерапии назначение базального инсулина в дополнение к терапии ПССП может быть эффективным для достижения целевых значений $\mathrm{HbA}_{1 c^{\prime}}$ однако со временем большинству пациентов требуется проведение интенсификации инсулинотерапии для поддержания оптимального гликемического контроля. В настоящее время при неэффективности базального инсулина могут быть использованы несколько вариантов стратегий интенсификации инсулинотерапии, такие как стратегия последовательного добавления прандиального инсулина по схеме базал плюс, назначение базис-болюсного режима или готовых смесей (двухфазного) инсулина. Все 3 стратегии обеспечивают эффективный контроль гликемии, демонстрируя безопасность и хорошую переносимость терапии. Однако каждый подход имеет свои преимущества и недостатки относительно риска развития гипогликемических реакций, влияния на динамику массы тела и разную степень сложности выполнения для пациента.

Стратегия базально-болюсной терапии является более сложным вариантом интенсификации инсулинотерапии, требующим дополнительного обучения пациентов самостоятельному управлению СД и высокой мотивации к проведению самоконтроля в сравнении с терапией двухфазными инсулинами. Готовые смеси инсулина - более простой вариант для пациентов с СД2, предполагает меньшее количество инъекций инсулина, более редкую частоту проведения самоконтроля гликемии, более простой алгоритм титрации; данная терапия может быть оптимальным вариантом для определенных категорий пациентов, кто не готов или не может по разным причинам выполнять все необходимые условия для проведения базально-болюсного режима инсулинотерапии.

Каждый режим терапии предлагает различные подходы и предоставляет клиницисту возможность адаптировать терапию с учетом индивидуальных целей гликемии, особенностей течения заболевания, а также потребностей пациента, в том числе пожеланий по количеству инъекций инсулина, частоте проведения самоконтроля гликемии и особенностей титрации дозы инсулина. Совместное обсуждение между врачом и пациентом тактики терапии позволит выбрать наиболее подходящий вариант для каждой конкретной клинической ситуации, повысить приверженность пациента к совместно выбранному режиму инсулинотерапии и обеспечить успех терапии.

\section{ДОПОЛНИТЕЛЬНАЯ ИНФОРМАЦИЯ}

Конфликт интересов. Светлана Елизарова и Абдул Джаббар - сотрудники компании Эли Лилли. Абдул Джаббар владеет акциями в Eli Lilly and Company. Е.В. Бирюкова декларирует отсутствие явных и потенциальных конфликтов интересов, связанных с публикацией данной статьи.

Финансовая поддержка. Часть исследований, представленных в данном обзоре, были спонсированы Eli Lilly and Company, производителем инсулинов Humulin ${ }^{\circledast}$ N, Humalog ${ }^{\circledast}$, Humalog ${ }^{\circledast}$ Mix75/25 $5^{\text {тм }}$ и Humalog ${ }^{\circledast}$ Miх50/50'м. Медицинская помощь в написании статьи представлена Julie A. Ely, PhD, CMPP и Rebecca Lew, PhD, CMPP of ProScribe - Envision Pharma Group, и финансировалась Eli Lilly and Company.

Участие авторов. С.В. Елизарова, А. Джаббар, Е.В. Бирюкова - подбор литературы, редактирование текста и одобрение окончательной версии рукописи.

\section{СПИСОК ЛИТЕРАТУРЫ | REFERENCES}

1. Дедов И.И., Шестакова М.В., Викулова О.К. Эпидемиология сахарного диабета в Российской Федерации: клинико-статистический отчет по данным Федерального регистра сахарного диабета // Сахарный диабет. - 2017. - Т. 20. - №1. - C. 13-41. [Dedov II, Shestakova MV, Vikulova OK. Epidemiology of diabetes mellitus in Russian Federation: clinical and statistical report according to the federal diabetes registry. Diabetes mellitus. 2017;20(1):13-41. (In Russ.)] doi: 10.14341/DM8664

2. IDF Diabetes Atlas, 7th edition. Brussels: International Diabetes Federation; 2015. Available from: http://www.diabetesatlas.org.
3. Дедов И.И., Шестакова М.В., Галстян Г.Р. Распространенность сахарного диабета 2 типа у взрослого населения России (исследование NATION) // Сахарный диабет. - 2016. - T. 19. - № 2. - C. 104-112. [Dedov II, Shestakova MV, Galstyan GR. The prevalence of type 2 diabetes mellitus in the adult population of Russia (NATION study). Diabetes Mellitus. 2016;19(2):104-112. (In Russ.)] doi: 10.14341/DM2004116-17

4. Litwak L, Goh SY, Hussein Z, et al. Prevalence of diabetes complications in people with type 2 diabetes mellitus and its association with baseline characteristics in the multinational A1 chieve study. Diabetol Metab Syndr. 2013;5(1):57-66. doi: 10.1186/1758-5996-5-57 
5. Sountsov YI, Dedov II, Shestakova MV, editors. Diabetes in Russia: Problems and Solutions. Moscow: Endocrinology Research Centre; 2008.

6. Holman RR, Paul SK, Bethel MA, et al. 10-year follow-up of intensive glucose control in type 2 diabetes. NEngl J Med. 2008;359(15):1577-1589. doi: 10.1056/NEJMoa0806470

7. UK Prospective Diabetes Study Group. Intensive blood-glucose control with sulphonylureas or insulin compared with conventional treatment and risk of complications in patients with type 2 diabetes (UKPDS 33). UK Prospective Diabetes Study (UKPDS) Group. Lancet. 1998;352(9131):837-853

8. Inzucchi SE, Bergenstal RM, Buse JB, et al. Management of hyperglycemia in type 2 diabetes: a patient-centered approach: position statement of the American Diabetes Association (ADA) and the European Association for the Study of Diabetes (EASD). Diabetes Care. 2012;35(6):1364-1379. doi: 10.2337/dc12-0413

9. American Diabetes Association. Cardiovascular Disease and Risk Management. Diabetes Care. 2017;40(Suppl 1):75-87. doi: $10.2337 / \mathrm{dc} 17-5012$

10. Дедов И.И., Шестакова М.В., Майоров А.Ю., и др. Алгоритмы специализированной медицинской помощи больным сахарным диабетом / Под редакцией И.И. Дедова, М.В. Шестаковой, А.Ю. Майорова. - 8-й выпуск // Сахарный диабет. - 2017. - Т. 20. №1S. - C. 1-121. [Dedov II, Shestakova MV, Mayorov AY, et al. Standards of specialized diabetes care. Edited by Dedov II, Shestakova MV, Mayorov AY. 8th edition. Diabetes mellitus. 2017;20(1S):1-121. (In Russ.)] doi: 10.14341/DM8146

11. Calvert MJ, MCManus RJ, Freemantle N. Management of type 2 diabetes with multiple oral hypoglycaemic agents or insulin in primary care: retrospective cohort study. Br J Gen Pract. 2007;57(539):455-460

12. Nichols GA, Gandra SR, Chiou C-F, et al. Successes and challenges of insulin therapy for type 2 diabetes in a managed-care setting. Curr Med Res Opin. 2010;26(1):9-15. doi: 10.1185/03007990903417679

13. Riddle MC, Rosenstock J, Gerich J; Insulin Glargine 4002 Study Investigators. The treat-to-target trial: randomized addition of glargine or human NPH insulin to oral therapy of type 2 diabetic patients. Diabetes Care. 2003;26(11):3080-3086. doi: 10.2337/diacare.26.11.3080

14. Mata-Cases M, Mauricio D, Franch-Nadal J. Clinical characteristics of type 2 diabetic patients on basal insulin therapy with adequate fasting glucose control who do not achieve $\mathrm{HbA1} \mathrm{c}$ targets. J Diabetes. 2017;9(1):34-44. doi: 10.1111/1753-0407.12373

15. Raskin $\mathrm{P}$, Allen E, Hollander P, et al. Initiating insulin therapy in type 2 diabetes: a comparison of biphasic and basal insulin analogs. Diabetes Care. 2005;28(2):260-265. doi: 10.2337/diacare.28.2.260

16. Gordon J, Pockett RD, Tetlow AP, et al. A comparison of intermediate and long-acting insulins in people with type 2 diabetes starting insulin: an observational database study. Int J Clin Pract. 2010;64(12):1609-1618. doi: 10.1111/j.1742-1241.2010.02520.x

17. Blak BT, Smith HT, Hards M, et al. A retrospective database study of insulin initiation in patients with Type 2 diabetes in UK primary care. Diabet Med. 2012;29(8):e191-198. doi: 10.1111/j.1464-5491.2012.03694.x

18. Polinski JM, Seoyoung KC, Dingfeng J, et al. Geographic patterns in patient demographics and insulin use in 18 countries, a global perspective from the multinational observational study assessing insulin use: understanding the challenges associated with progression of therapy (MOSA1c). BMC Endocr Disord. 2015;(15):46. doi: 10.1186/s12902-015-0044-z
19. Patrick AR, Fischer MA, Choudhry NK, et al. Trends in insulin initiation and treatment intensification among patients with type 2 diabetes. $J$ Gen Intern Med. 2014;29(2):320-327. doi: 10.1007/s1 1606-013-2643-6

20. Khunti S, Davies MJ, Khunti K. Clinical inertia in the management of type 2 diabetes mellitus: a focused literature review. Br J Diabetes Vasc Dis. 2015;15(2):65-69. doi: 10.15277/bjdvd.2015.019

21. Owens DR, van Schalkwyk C, Smith P, et al. Algorithm for the introduction of rapid-acting insulin analogues in patients with type 2 diabetes on basal insulin therapy. Practical Diabetes. 2009;26(2):70-77. doi: 10.1002/pdi.1339

22. Rodbard HW, Visco VE, Andersen $\mathrm{H}$, et al. Treatment intensification with stepwise addition of prandial insulin aspart boluses compared with full basal-bolus therapy (FullSTEP Study): a randomised, treatto-target clinical trial. Lancet Diabetes Endocrinol. 2014;2(1):30-37. doi: 10.1016/S2213-8587(13)70090-1

23. Rosenstock J, Ahmann AJ, Colon G, et al. Advancing insulin therapy in type 2 diabetes previously treated with glargine plus oral agents: prandial premixed (insulin lispro protamine suspension/lispro) versus basal/bolus (glargine/lispro) therapy. Diabetes Care. 2008;31(1):20-25. doi: $10.2337 /$ dc07-1122

24. Edelman SV, Liu R, Johnson J, Glass LC. AUTONOMY: the first randomized trial comparing two patient-driven approaches to initiate and titrate prandial insulin lispro in type 2 diabetes. Diabetes Care. 2014:37(8):2132-2140. doi: 10.2337/dc13-2664

25. Rodbard HW, Karolicki B. Management of type 2 diabetes - methods for addition of prandial to basal insulin. European Endocrinology. 2014;10(2):124-130. doi: 10.17925/EE.2014.10.02.124

26. Latif ZA, Hussein Z, Litwak L, et al. Safety and effectiveness of insulin aspart in basal-bolus regimens regardless of age: A1chieve study results. Diabetes Ther. 2013;4(1):103-118. doi: 10.1007/s13300-013-0023-1

27. Harris SB, Yale JF, Berard L, et al. Does a patient-managed insulin intensification strategy with insulin glargine and insulin glulisine provide similar glycemic control as a physician-managed strategy? Results of the START (Self-Titration With Apidra to Reach Target) study: a randomized noninferiority trial. Diabetes Care. 2014;37(3):604-610. doi: $10.2337 / \mathrm{dc} 13-1636$

28. Ilag LL, Kerr L, Malone JK, Tan MH. Prandial premixed insulin analogue regimens versus basal insulin analogue regimens in the management of type 2 diabetes: an evidence-based comparison. Clin Ther. 2007;29(6 Pt 1):1254-1270. doi: 10.1016/j.clinthera.2007.07.003

29. Tinahones FJ, Gross JL, Onaca A, et al. Insulin lispro low mixture twice daily versus basal insulin glargine once daily and prandial insulin lispro once daily in patients with type 2 diabetes requiring insulin intensification: a randomized phase IV trial. Diabetes Obes Metab. 2014;16(10):963-970. doi: 10.1111/dom.12303

30. Giugliano D, Sieradzki J, Stefanski A, Gentilella R. Personalized intensification of insulin therapy in type 2 diabetes - does a basal-bolus regimen suit all patients? Curr Med Res Opin. 2016;32(8):1425-1434. doi: 10.1080/03007995.2016.1181051

31. Dieuzeide G, Chuang L-M, Almaghamsi A, et al. Safety and effectiveness of biphasic insulin aspart 30 in people with type 2 diabetes switching from basal-bolus insulin regimens in the A1 chieve study. Prim Care Diabetes. 2014;8(2):111-117. doi: 10.1016/j.pcd.2013.07.005

32. Mathieu C, Storms F, Tits J, et al. Switching from premixed insulin to basal-bolus insulin glargine plus rapid-acting insulin: the ATLANTIC study. Acta Clin Belg. 2013;68(1):28-33. doi: 10.2143/ACB.68.1.2062716

\section{ИНФОРМАЦИЯ ОБ АВТОРАХ [AUTHORS INFO]}

Елизарова Светлана Викторовна [Svetlana V. Elizarova, MD]; адрес: 123317, Москва, Пресненская набережная, д. 10 [address: 10, Presnenskaya naberezhnaya, Moscow, 123317 Russian Federation];ORCID: http://orcid.org/0000-0001-5001-306X; eLibrary SPIN: 1176-5255; e-mail:elizarova_svetlana@lilly.com Бирюкова Елена Валерьевна, д.м.н., профессор [Elena V. Biryukova, MD, PhD]; ORCID: http://orcid.org/0000-0001-9007-4123; eLibrary. SPIN: 3700-9150; e-mail: lena@obsudim.ru. Джаббар Абдул [Abdul Jabbar, MD]; ORCID: http://orcid.org/0000-0002-6380-2225; e-mail: jabbar_abdul@lilly.com.

\section{ЦИТИРОВАТЬ:}

Бирюкова Е.В., Джаббар А., Елизарова С.В. Когда базальный инсулин неэффективен: стратегии интенсификации инсулинотерапии у пациентов с сахарным диабетом 2 типа // Сахарный диабет. — 2017. — Т. 20. — №5. — C. 363-373. doi: 10.14341/DM8824

\section{TO CITE THIS ARTICLE:}

Biryukova EV, Jabbar A, Elizarova SV. When basal insulin is not enough: successful strategies for insulin intensification in patients with type 2 diabetes mellitus. Diabetes mellitus. 2017;20(5):363-373. doi: 10.14341/DM8824 